\title{
Third-Generation Nephrectomy by Natural Orifice Transluminal Endoscopic Surgery
}

\author{
Estevao Lima, Carla Rolanda, José M. Pêgo, Tiago Henriques-Coelho, David Silva, Luís Osório, \\ Ivone Moreira, José L. Carvalho and Jorge Correia-Pinto* \\ From the Life and Health Sciences Research Institute, School of Health Sciences, University of Minho (EL, CR, JMP, DS, JCP) and \\ Departments of Gastroenterology and Anesthesiology, S. Marcos Hospital (CR, JMP), Braga, Department of Urology, St. António General \\ Hospital (EL, LO) and Department of Pediatric Surgery, S. Joao Hospital (THC, JLC, JCP), Porto and Department of Oncology, Sra. \\ Oliveira Hospital (IM), Guimarães, Portugal
}

\begin{abstract}
Purpose: Recently there has been increasing enthusiasm for performing simple abdominal procedures by transgastric surgery. We previously reported the usefulness of a combined transgastric and transvesical approach to cholecystectomy. In this study we assessed the feasibility of combined transgastric and transvesical approach for performing a more complex surgical procedure, such as nephrectomy, in a porcine model.

Materials and Methods: In a nonsurvival study combined transgastric and transvesical approaches were established in 6 female pigs. Under ureteroscope guidance we installed a transvesical $5 \mathrm{~mm}$ over tube into the peritoneal cavity and a flexible gastroscope was passed orally into the peritoneal cavity by a gastrotomy. We performed right or left nephrectomy with instruments introduced by the 2 devices that worked in the renal hilum, alternating device intervention for dissection and retraction procedures.

Results: Four right and 2 left nephrectomies were performed. There were no complications during the creation of transvesical and transgastric access. In all animals we visualized the 2 kidneys. The renal vessels and ureter were reasonably individualized and ligated separately with ultrasonic scissors, which were introduced through the transvesical port. In 2 early cases mild hemorrhage occurred after ultrasonic ligation. To overcome this complication we applied clips successfully before ultrasonic ligation in the remaining animals. Thus, complete renal release and mobilization to the stomach were achieved in all animals.
\end{abstract}

Conclusions: Nephrectomy by natural orifices using the combined transgastric and transvesical approach is technically feasible, although to our knowledge there is no reliable method for removing the specimen with current instruments.

Key Words: kidney; swine; nephrectomy; endoscopy; surgical procedures, minimally invasive

$\mathrm{R}$ enal surgery has its origin some 400 years B.C.E. with the drainage of abscesses and the removal of calculi from renal fistulas. In the early 19th century kidneys were sometimes removed inadvertently during attempted ovarian surgery with the observation that the remaining kidney continued to produce normal amounts of urine. However, it was not until 1869 that Simon performed the first planned nephrectomy. ${ }^{1}$ During the last century there was progressive development of the surgical technique, aiming mainly at organ resection without apprehension and associated morbidity.

With the first laparoscopic nephrectomy in 1990 performed by Clayman et al a revolution began with the implementation of laparoscopic techniques that had become ac-

Submitted for publication February 13, 2007.

Study received approval from ethical review boards at Minho University, Braga, Portugal.

Supported by Bolsa de Investigação Básica JABA 2006 da Associação Portuguesa de Urologia, Bolsa de Investigação da Sociedade Portuguesa de Endoscopia Digestiva 2006 and Grant POCI/SAUOBS/56428/2004 from FCT-Portugal.

* Correspondence: Instituto de Ciências da Vida e Saúde, Escola de Ciências da Saúde, Universidade do Minho, Campus de Gualtar, 4709-057 Braga, Portugal (telephone: + 351253604 872; FAX: + 351 253604 831; e-mail: jcp@ecsaude.uminho.pt). cepted by the urological community worldwide, initially for benign and more recently for malignant renal disease. ${ }^{2}$ The main reasons that minimally invasive surgery increased in popularity were the many proven advantages over traditional open procedures, such as minimal scarring, decreased pain and more rapid patient recovery. ${ }^{3}$

Currently NOTES is being studied as a potentially less invasive alternative to conventional laparoscopy for intraabdominal surgery. In fact, there is increasing hope that we will be able to perform the most common abdominal procedures in humans using this revolutionary technique that seems to be third-generation surgery. After the development of transvaginal peritoneal access, mainly for specimen extraction, ${ }^{4}$ Gettman et al used this approach to perform nephrectomy. ${ }^{5}$ More recently transgastric access to the peritoneal cavity was described with unexpected success. ${ }^{6}$ Subsequently we had the opportunity to test the feasibility and safety of a transvesical port to the peritoneal and thoracic cavities. ${ }^{7,8}$ This port was revealed to be particularly important because some procedures that appeared hazardous and not viable using an isolated transgastric port become feasible and safe when performed by a combined transgastric and transvesical approach, as we recently described for cholecystectomy. ${ }^{9}$ 
Before translation in humans additional preclinical studies are still needed to increase our confidence with these techniques in high risk procedures such as nephrectomy. ${ }^{10}$ We report the feasibility of the combined transgastric and transvesical approach for performing scarless natural orifice nephrectomy in a porcine model.

\section{MATERIALS AND METHODS}

This study was approved by ethical review boards at Minho University, Braga, Portugal. After a surgical learning curve of 4 animals (data not shown) right or left nephrectomy was performed in 6 consecutive anesthetized female pigs (Sus scrofus domesticus) weighing 25 to $30 \mathrm{~kg}$. After the surgical procedures the animals were immediately sacrificed and necropsy was performed.

\section{Pig Preparation}

The animals were fed liquids for 3 days and then were denied food for 24 hours and water for 6 before surgical intervention. All procedures were performed using general anesthesia, as described previously. ${ }^{7}$

\section{Surgical Technique and Instruments}

The technique of performing nephrectomy by NOTES was begun using a transvesical port and subsequently a transgastric port (fig. 1). Through the transvesical port we used a rigid Olympus® A2942A ureteroscope, LCSC5L UltraCision® Harmonic Scalpel® Long Shears ultrasonic scissors or an EL5ML Ligamax ${ }^{\mathrm{TM}} 5$ clip applicator. Through the transgastric port an adult, forward viewing, double channel Olympus GIF-2T160 endoscope was introduced. Through the working channels of the 2 endoscopes we used certain instruments, including 1) ureteroscope instruments (Olympus A2574 grasping forceps and Olympus A2576 scissors) and 2) gastroscope instruments (an Olympus KD-11Q-1 needle knife, a Microvasive ${ }^{\circledR}$ 5156-01 guidewire, a Microvasive 5837 through the scope balloon, Olympus FG-6L-1 and FG47L-1 grasping forceps, a KD-16Q-1 papillotomy knife and a Sensation $^{\mathrm{TM}}$ M00562650 endoscopic snare). For cautery we used standard Olympus PSD 20 electrocautery equipment.

\section{Transvesical Access}

A transvesical port was established, as previously described by our group. ${ }^{7}$ Briefly, a ureteroscope was introduced into the bladder with $\mathrm{CO}_{2}$ distention. After making a small mucosal incision on the bladder dome we used a $5 \mathrm{Fr}$ open end 62450200 Selectip ${ }^{\mathrm{TM}}$ ureteral catheter to perform cystotomy. Guided by a 0.035-inch flexible tip RF*GA35153M Terumo® guidewire the vesical hole was enlarged with the dilator of a 250-105 Microvasive ureteroscope sheath, which was enveloped with a 5.5 $\mathrm{mm}$ over tube. A rigid ureteroscope was introduced into the peritoneal cavity within the over tube, allowing the creation of pressure controlled $\mathrm{CO}_{2}$ pneumoperitoneum as necessary. The peritoneal cavity was thoroughly examined.

\section{Transgastric Access}

As previously described, ${ }^{9}$ we introduced the gastroscope into the peritoneal cavity through a gastrotomy established on the anterior stomach wall. The gastric wall incision was made by a needle knife with cautery and it was then increased using a papillotomy knife. All procedures were mon-

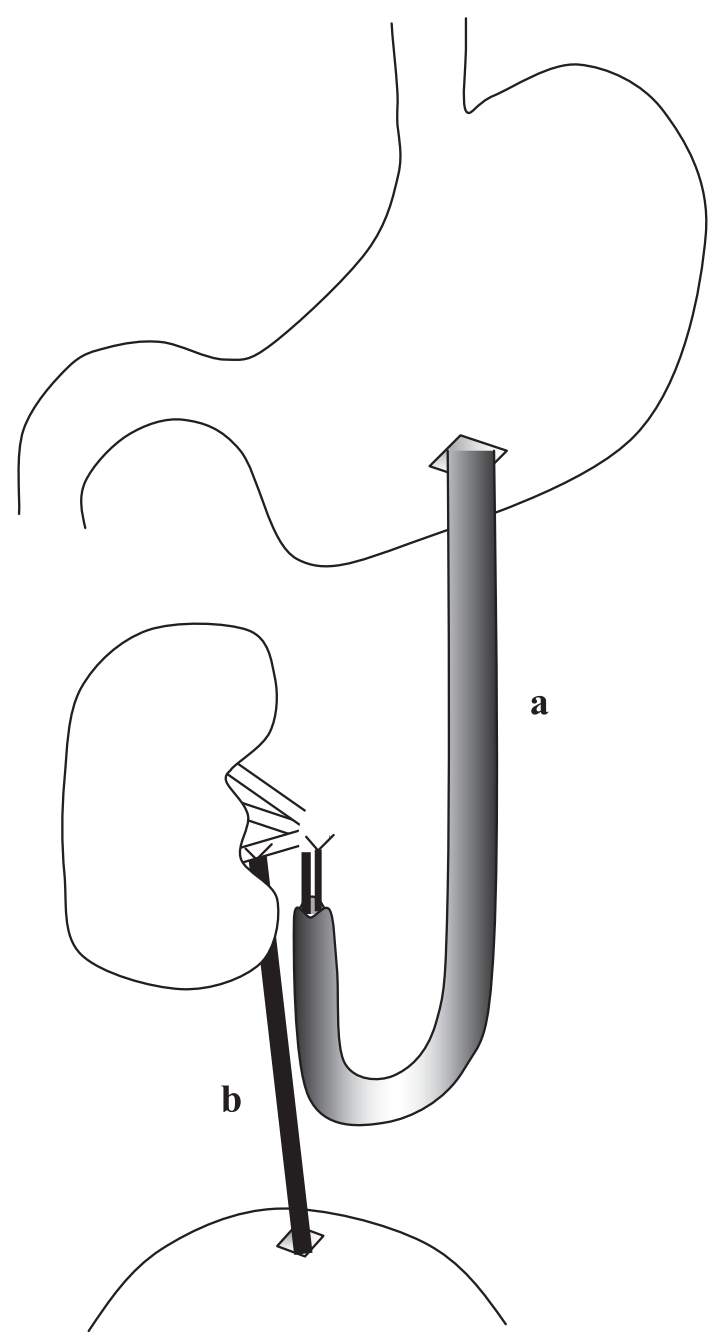

FiG. 1. Instrument positioning for nephrectomy by combined transgastric and transvesical approach. $a$, gastroscope in retroflexion approaching renal hilus. $b$, ureteroscope.

itored using the view provided by the ureteroscope introduced through the transvesical port.

\section{Nephrectomy Procedure}

The animals were placed in the lateral decubitus position at the beginning of the procedure to expose the contralateral kidney. Subsequently the selected kidney and respective hilum were immediately identified by the 2 endoscopes. We then mobilized the lower kidney pole by opening the parietal peritoneum with cautery using the needle knife introduced by the gastroscope. This procedure was helped by suspending the peritoneum using grasping forceps introduced through the working channel of the ureteroscope. Subsequently the peritoneum was reflected off the kidney hilum by serial combined actions of the grasping forceps and a needle knife introduced through the ureteroscope and gastroscope, respectively. This maneuver exposed the hilar elements, such as the renal vein, renal artery and urinary excretory structure. The renal vessels were then individualized and completely dissected from the surrounding tissues using instruments introduced through the 2 endoscopes, which worked in coordinated fashion. 
After the renal artery and vein were completely dissected and circumferentially individualized the ureteroscope was removed from the peritoneal cavity. Subsequently $5 \mathrm{~mm}$ ultrasonic scissors were introduced into the peritoneal cavity through the transvesical over tube and guided to the renal hilum using the gastroscopic image. The artery was positioned between the blades of the ultrasonic scissors and divided using level 1 for maximum coagulation. The renal vein was divided in a similar procedure. In 2 pigs the renal vessels were clipped before division by the ultrasonic scissors. It is worth mention that the renal vessels were divided using $5 \mathrm{~mm}$ transvesical instruments, which were used under gastroscope guidance. When the renal hilum was completely free, we completed kidney dissection by isolating and mobilizing the upper pole using transvesical ultrasonic scissors or the needle knife, always under gastroscope guidance. Finally, we divided the ureter by half of its trajectory and the kidney was dragged from its bed in the direction of the stomach, held by an endoscopic snare. However, the kidney was left in the animal since we were not able to extract it with the current instruments.

After removing the organ the hilar renal area was washed and inspected for bleeding. Adjacent organs were evaluated for evidence of laceration and perforation.

\section{RESULTS}

Nephrectomy by the combined transgastric and transvesical approach was done in 6 pigs. The procedures involved in the creation of the vesical hole (cystoscopy, bladder mucosal incision, cystotomy and transvesical over tube passage) were performed easily and rapidly, and without complications in all animals. The ureteroscope was introduced in straightforward fashion into the peritoneal cavity and $\mathrm{CO}_{2}$ insufflation was performed without incident. The ureteroscopic image was particularly valuable for helping the gastroscope operator select the most appropriate point for gastrotomy on the anterior gastric wall (fig. 2). Additionally, the external view of the gastric wall provided by the ureteroscope allowed us to create the gastrotomy while preventing damage to the major gastric vessels and adjacent structures (fig. $2, B$ ). In fact, beginning access to the peritoneal cavity through the transvesical approach resulted in no complications during gastric incision and gastroscope entrance into the peritoneal cavity.

From the transgastric and transvesical ports it was possible to easily find the selected kidney for nephrectomy
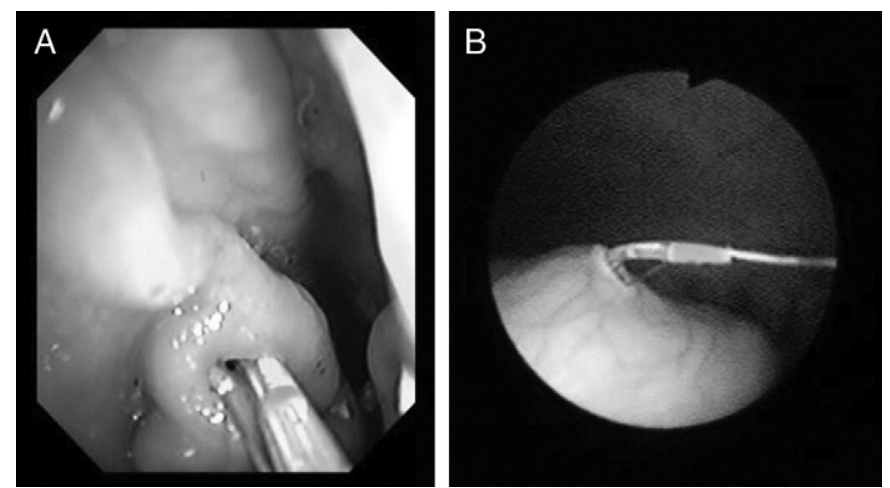

FIG. 2. Gastrotomy creation with papillotomy knife. A, gastroscopic image shows internal view. $B$, ureteroscopic image shows external view.
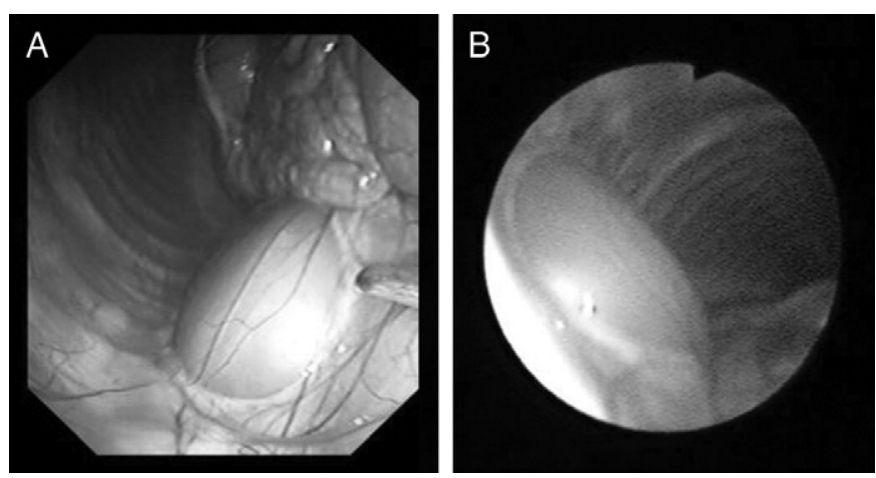

Fig. 3. Renal identification before starting dissection. $A$, gastroscopic image. $B$, ureteroscopic image.

(fig. 3). It should be stressed that the gastroscope was used most of the time in retroflexion. In fact, the kidney was more easily visualized by the gastroscope in the retroflexion position, directing its tip toward the upper quadrant. This allowed us to work with the gastroscope in a stable position.

Further procedures in the performance of nephrectomy were done using coordinated movements of the gastroscope and ureteroscope operators, which increased during the experimental protocol. Creation of a peritoneal window in the lower pole of the kidney and subsequent dissection to expose the renal hilum were accomplished in all animals in a rapid and safe way (fig. 4). These procedures were done most of the time by the gastroscope operator, whereas the ureteroscope operator grasped the peritoneum. Maneuvers to dissect and isolate the hilar vessels were done using gastroscope or ureteroscope instruments, always in coordinated movements (fig. 5). Although it was time-consuming, vessel isolation was reasonably accomplished without hemorrhage in all animals.

To promote renal vessel ligation we always used the same sequence, characterized by removal of the ureteroscope and followed by the introduction of ultrasonic scissors into the peritoneal cavity through the transvesical over tube. Transvesical instrument exchange was always guided by the gastroscopic image (fig. 6). In the first 2 animals ligation of the artery and vein with the ultrasonic scissors was efficient with no evidence of hemorrhage (figs. 7 and 8). In animals 3 and 4 ultrasonic ligation of the renal artery was insufficient, causing mild hemorrhage that significantly blurred the view of renal hilar structures. Although in these cases hemorrhage could be safely controlled with gastroscope instru-
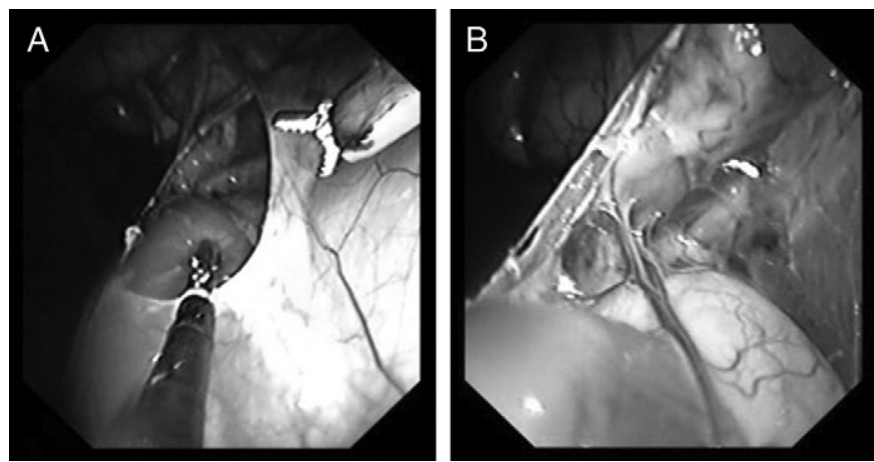

Fig. 4. Creation of peritoneal window for hilar approach. A, opening peritoneum. $B$, visualizing hilar elements. 

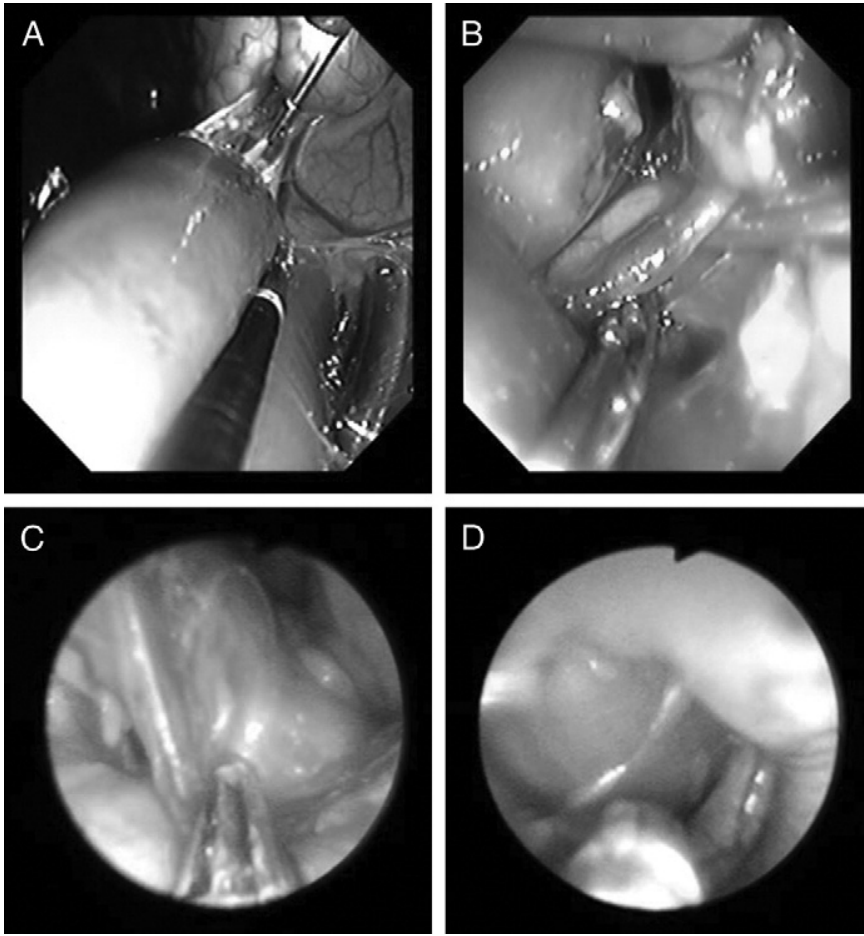

FIG. 5. Renal vessel dissection. $A$, gastroscopic view of renal vein. $B$, gastroscopic view of renal artery. $C$, ureteroscopic view of renal artery. $D$, ureteroscopic view of renal vein.

ments, such as grasping forceps followed by ultrasonic cautery reapplication, in the last 2 animals we successfully applied endoscopy clips before ultrasonic ligation to increase the safety of vessel ligation. Clip application was easy and

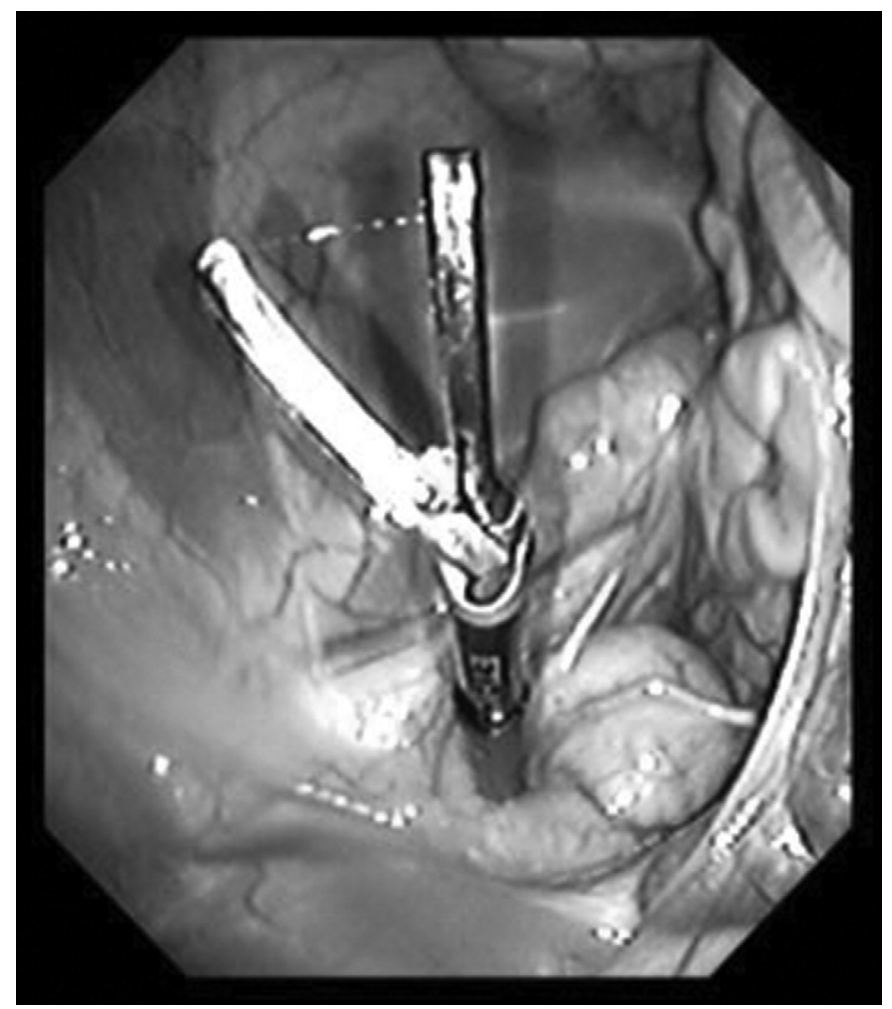

FiG. 6. Gastroscopic view of entrance of ultrasonic scissors into abdomen by transvesical port.
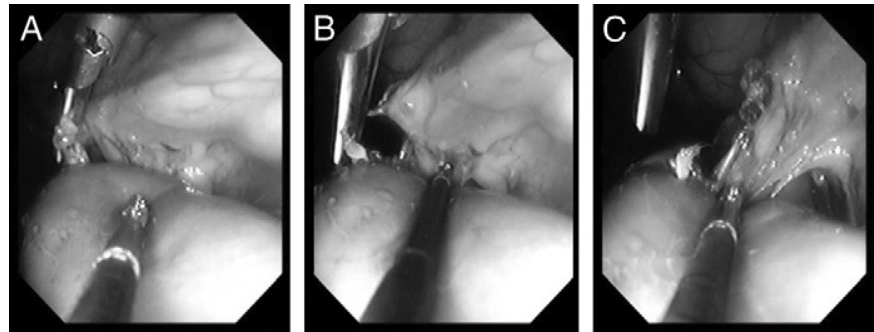

FIG. 7. Gastroscopic image reveals renal artery ultrasonic ligation. $A$, starting ligation. $B$, finishing ligation. $C$, completed ligation.

this approach was particularly successful with hemorrhage in none of these cases.

After vessel ligation ureteral dissection and section were easily accomplished in all animals (fig. 9). The kidney was easily released by sectioning the peritoneum from the upper renal pole and posterior perirenal tissue. For these procedures we used gastroscope cautery or transvesical ultrasonic scissors. The kidney was then mobilized from the renal bed, held with a gastroscopic snare and pulled to the stomach (fig. 10).

Nephrectomy was performed under pressure controlled $\mathrm{CO}_{2}$ pneumoperitoneum. However, it should be emphasized that most of the time the surgical procedure was performed under a low $\mathrm{CO}_{2}$ pressure of around $3 \mathrm{~mm} \mathrm{Hg}$. In fact, pneumoperitoneal pressure was increased up to $12 \mathrm{~mm} \mathrm{Hg}$ only during gastric perforation and exchange of the transvesical surgical instrument.

Median time for the overall procedure, including establishment of the transvesical and transgastric port, was around 120 minutes (range 90 to 150 ). Procedure time decreased with experience.

All animals were sacrificed at the end of the nephrectomy procedure, immediately after surgery. Necropsy did not reveal any damage to the abdominal viscera that was related to transgastric and transvesical access to the peritoneal cavity. It was possible to confirm nephrectomy and perfect ligation of the renal vessels.

\section{DISCUSSION}

The current study confirms that nephrectomy is feasible exclusively by NOTES. Moreover, the combined transgastric and transvesical approach was particularly useful for complete renal manipulation. Thus, renal intervention might be included in the list of potential clinical indications for NOTES.

This study describes a revolutionary surgical approach that is being used for an increasing number of procedures, such as
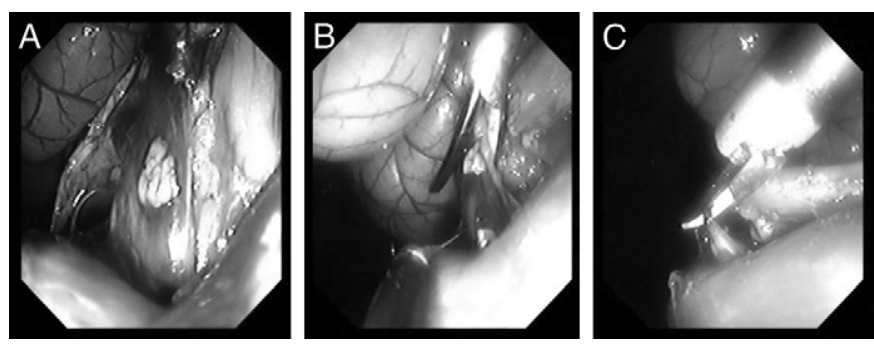

FIG. 8. Gastroscopic image demonstrates renal vein ultrasonic ligation. $A$, dissected vein. $B$, starting ligation. $C$, finishing ligation. 

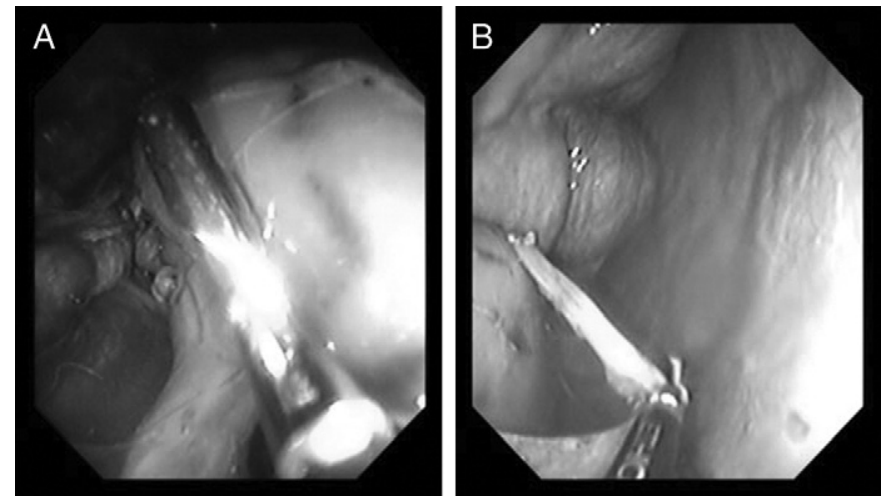

FIG. 9. $A$, individualized ureter. $B$, ureteral ligation

fallopian tube ligation, cholecystectomy, gastrojejunostomy, partial hysterectomy, oophorectomy and splenectomy essentially via the transgastric route. ${ }^{11-17}$ In fact, there are several theoretical advantages to NOTES. 1) There are no abdominal incisions and, therefore, abdominal wound infections and incisional hernias are avoided, possibly resulting in less pain and certainly in a better cosmetic effect. 2) There may be potential advantages of a more rapid recovery, fewer adhesions and less postoperative ileus. 3) The natural orifice approach to the peritoneal cavity may be the ideal route in morbidly obese patients. ${ }^{10}$

Believing in the potential benefits of natural orifices approach to abdominal surgery, to our knowledge we were the first group to use a combined transgastric and transvesical approach to perform cholecystectomy, aiming to overcome many limitations that were previously described for the isolated transgastric approach related to anatomy exposure, organ retraction, grasping and limited triangulation. ${ }^{9}$ Interestingly the combination of transgastric and transvesical ports was also particularly useful for approaching the kidney, making nephrectomy feasible by NOTES. We used the transvesical port because it is diametrically opposed to the transgastric port and seems to offer additional advantages over transvaginal and transcolonic ports. ${ }^{18}$ In fact, although the transvesical port does not support as large instruments as transvaginal and transcolonic ports, it is sterile, available in the 2 genders and seems particularly safe, at least in a porcine model, even when left unclosed. Moreover, the bladder dome offers the most anterior position in the lower abdomen, allowing the introduction of surgical instruments above the bowel loops. ${ }^{7,19}$ As proven by Gettman et al, ${ }^{5}$ the great advantage of the transvaginal approach is its availability to remove the specimen.

Regarding the surgical technique, after a short learning curve we could open the renal peritoneum and dissect the renal vessels and ureter in a safe way. To open the peritoneum and dissect the hilum the coordination of the gastroscope and ureteroscope operators was vital. In fact, the 2 operators were constantly alternating their intervention on dissection vs retraction procedures. Whereas the ureteroscope had the advantage of being rigid, it had a significant limitation in width and image resolution as well as its fragile instruments. In contrast, the gastroscope had an enormous advantage in width and image quality but its flexibility and unstable platform frequently compromised its intervention. The gastroscope operator dealt with this limitation, working frequently in retroflexion with the gastro- scope loop supported on the abdominal walls. With this approach we could dissect the right or left kidney in a similar way. However, it might be emphasized that in pigs the 2 kidneys are not hidden by the colon. In fact, predicting translation into humans, we should not neglect that the colon loop might complicate any renal approach by NOTES.

Regarding vessel ligation, we refused the idea of using an endoscopic loop to ligate the renal vein and artery simultaneously, as others described for splenectomy. ${ }^{17}$ We chose to individualize each vessel, which we reasonably accomplished in the majority of cases, ligating them individually with ultrasonic scissors. Ultrasonic ligation was sufficient for most vessels but in 2 animals we observed mild hemorrhage after ultrasonic ligation. In this sequence we successfully applied surgical clips in some animals before ultrasonic ligation. The ureter was also easily dissected and ligated in all cases. After complete kidney release we used an endoscopic snare to hold up the organ and pull it out to the stomach.

During our experiments we realized that most of the time we could work safely with a low $\mathrm{CO}_{2}$ pressure of approximately $3 \mathrm{~mm} \mathrm{Hg}$. We have 2 major explanations for this finding. 1) The entrance of the instruments into the abdomen is parallel to the abdominal wall. Thus, we do not need significant pneumoperitoneum because the instruments use the abdominal wall as a fulcrum when mobilized. 2) The close-up properties of endoscopic instruments, particularly the gastroscope, might be particularly relevant because, if confirmed in posterior experiments, it could mean that the surgical stress of NOTES procedures might be significantly decreased compared with that of laparoscopy. In fact, most surgical laparoscopic stress is related to $\mathrm{CO}_{2}$ pressure. ${ }^{20}$

A major limitation of the current technique is related to our inability to safely close the gastrotomy. In fact, a critical element of any transgastric procedure is the ability to securely close the gastrotomy site that is required for endoscope passage and specimen removal. It is generally considered that an appreciable increase in patient morbidity from postoperative gastric leaks would expunge any patient advantage of the transgastric approach. For NOTES to achieve widespread adoption gastrotomy closure must be completely reliable. In this regard there are currently several endoscopic suturing devices in development. ${ }^{21}$ Because we do not have available at our laboratory a reliable device to close the gastrotomy, we did not enlarge the gastrotomy to pull the specimen into the stomach. To promote its extraction we
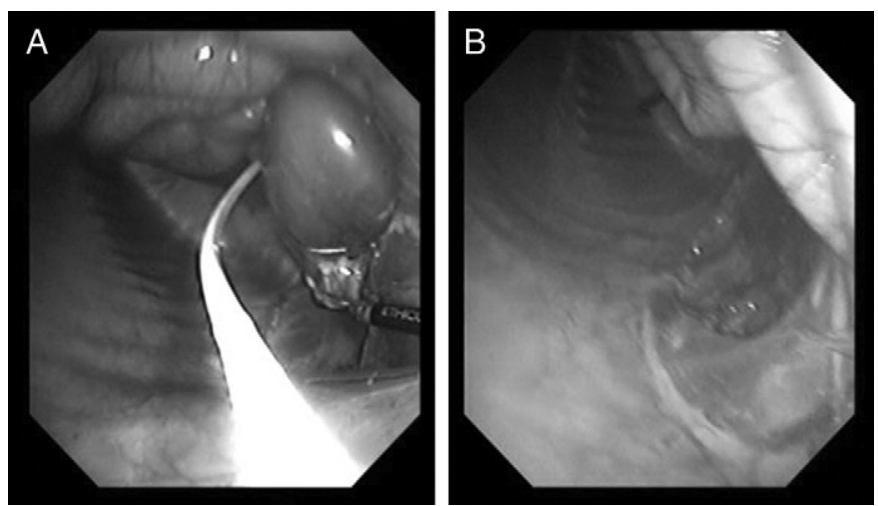

Fig. 10. Completed nephrectomy. $A$, gastroscopic snare drags released kidney. $B$, renal bed after kidney removal. 
predicted that it would be removed after its division or morcellation. Thus, we decided to sacrifice all animals after nephrectomy and necropsy revealed that the renal vessels were completely sealed.

A common drawback of the NOTES approach is its limited capability to deal with perioperative complications. Although we were able to control mild hemorrhage with current commercial instruments, we believe that new instruments and devices are needed to increase our confidence in NOTES to perform complex intra-abdominal surgical procedures. Current ureteroscopes are highly developed for diagnostic and limited therapeutic tasks in the urinary tract but they are far from the ideal design to be used in NOTES. They have several limitations that limit their capabilities. 1) The image quality of the ureteroscope is not similar to that of the gastroscope or laparoscope and light intensity is also sometimes inadequate. 2) Ureteroscopes usually have a diameter of between 3.3 and $4.3 \mathrm{~mm}$ with 1 or 2 working channels of $4.2 \mathrm{Fr}$ and $6.6 \mathrm{Fr}$, respectively, which limits the size of instruments. The endoscope shaft should be $5 \mathrm{~mm}$ and it should contain a larger channel to introduce other instruments with better efficiency. 3) Although the current rigid ureteroscope has some advantage for NOTES, such as allowing vigorous organ retraction for exposure, its rigidity might be a limitation to achieve retroperitoneum and other organs that are not in the axis of the bladder dome. Moreover, the tip of the ureteroscope should have flexibility and the ability to maneuver in all planes, allowing better tissue manipulation. The ideal device should allow complete rigidity for insertion and positioning with subsequent rigidity of the shaft, allowing traction/counter traction and continued flexibility of the tip, which should free the surgeon hands to manipulate different organs and tissues. 4) We should emphasize that the ultrasonic shears and even the clips that we used in this study for hilar ligation are not consensually approved for human purposes.

Although these concepts of NOTES could seem futuristic, we believe that guidelines for NOTES have already been established. Moreover, robotics and magnetic positioning technology can provide additional input for NOTES. ${ }^{22}$ The feasibility of nephrectomy by NOTES appears in our understanding as the extreme of a large spectrum of renal procedures that can potentially be done by NOTES in the near future. However, we might consider that much study is still needed to refine techniques, verify safety and document efficacy before translation into humans to minimize unexpected complications.

\section{CONCLUSIONS}

Right and left nephrectomy using NOTES was feasible in a porcine model. Our study also demonstrates the limitations of the standard devices since we could not reliably achieve gastrotomy closure and remove the specimen. This study provides encouragement to further innovative programs to create devices designed to advance the safety of NOTES.

\section{ACKNOWLEDGMENTS}

Paulo Pereira and José Bragança, Ethicon Endo-Surgery, Portugal and Alexandre Rocha, Olympus Portugal provided assistance.

\section{Abbreviations and Acronyms}

NOTES $=$ natural orifice transluminal endoscopic surgery

\section{REFERENCES}

1. Moll F and Rathert P: The surgeon and his intention: Gustav Simon (1824-1876), his first planned nephrectomy and further contributions to urology. World J Urol 1999; 17: 162.

2. Clayman RV, Kavoussi LR, Soper NJ, Dierks SM, Meretyk S, Darcy MD et al: Laparoscopic nephrectomy: initial case report. J Urol 1991; 146: 278.

3. Harrell AG and Heniford T: Minimally invasive abdominal surgery: lux et veritas past, present, and future. Am J Surg 2005; 190: 239.

4. Gill IS, Cherullo EE, Meraney AM, Borsuk F, Murphy DP and Falcone T: Vaginal extraction of the intact specimen following laparoscopic radical nephrectomy. J Urol 2002; 167: 238.

5. Gettman MT, Lotan Y, Napper CA and Cadeddu JA: Transvaginal laparoscopic nephrectomy: development and feasibility in the porcine model. Urology 2002; 59: 446.

6. Kalloo AN, Singh VK, Jagannath SB, Niiyama H, Hill SL, Vaughn CA et al: Flexible transgastric peritoneoscopy: a novel approach to diagnostic and therapeutic interventions in the peritoneal cavity. Gastrointest Endosc 2004; 60: 114.

7. Lima E, Rolanda C, Pêgo JM, Henriques-Coelho T, Silva D, Carvalho JL et al: Transvesical endoscopic peritoneoscopy: a novel $5 \mathrm{~mm}$-port for intra-abdominal scarless surgery. J Urol 2006; 176: 802.

8. Lima E, Henriques-Coelho T, Rolanda C, Pego JM, Silva D, Carvalho JL et al: Transvesical thoracoscopy: a natural orifice transluminal endoscopic approach for thoracic surgery. Surg Endosc 2007; 21: 854.

9. Rolanda C, Lima E, Pego JM, Henriques-Coelho T, Silva D, Carvalho JL et al: Third generation cholecystectomy by natural orifices: transgastric and transvesical combined approach (with video). Gastrointest Endosc 2007; 65: 111.

10. Rattner D and Kalloo A: ASGE/SAGES Working Group on natural orifice transluminal endoscopic surgery. SAGES/ ASGE Working Group on NOTES. Surg Endosc 2006; 20: 329.

11. Jagannath SB, Kantsevoy SV, Vaughn CA, Chung SSC, Cotton PB, Gostout CJ et al: Per-oral transgastric ligation of fallopian tubes with long-term survival in a porcine model. Gastrointest Endosc 2005; 61: 449.

12. Park PO, Bergstrom M, Ikeda K, Fritscher-Ravens A and Swain P: Experimental studies of transgastric gallbladder surgery: cholecystectomy and cholecystogastric anastomosis (videos). Gastrointest Endosc 2005; 61: 601.

13. Kantsevoy SV, Jagannath SB, Niiyama H, Chung SSC, Cotton PB, Gostout CJ et al: Endoscopic gastrojejunostomy with survival in a porcine model. Gastrointest Endosc 2005; 62: 287.

14. Bergstrom M, Ikeda K, Swain P and Park PO: Transgastric anastomosis by using flexible endoscopy in a porcine model. Gastrointest Endosc 2006; 63: 307.

15. Wagh MS, Merrifield BF and Thompson CC: Survival studies after endoscopic transgastric oophorectomy and tubectomy in a porcine model. Gastrointest Endosc 2006; 63: 473.

16. Merrifield BF, Wagh MS and Thompson CC: Peroral transgastric organ resection in the Abdomen: feasibility study in pigs. Gastrointest Endosc 2006; 63: 693.

17. Kantsevoy SV, Hu B, Jagannath SB, Vaughn CA, Beitler DM, Chung SSC et al: Transgastric endoscopic splenectomy. Is it possible? Surg Endosc 2006; 20: 522. 
18. Fong DG, Pai RD and Thompson CC: Transcolonic endoscopic abdominal exploration: a NOTES survival study in a porcine model. Gastrointest Endosc 2007; 65: 312.

19. Rolanda C, Lima E and Correia-Pinto J: Searching the best approach for third-generation cholecystectomy. Gastrointest Endosc 2007; 65: 354.

20. Neudecker J, Sauerland S, Neugebauer E, Bargamaschi R, Bonjer HJ, Cuschieri A et al: European Association for Endoscopic Surgery clinical practice guideline on the pneumoperitoneum for laparoscopic surgery. Surg Endosc 2002; 16: 1121.

21. Sclabas GM, Swain P and Swanstrom LL: Endoluminal methods for gastrotomy closure in natural orifice transenteric surgery (NOTES). Surg Innov 2006; 13: 23.

22. Park S, Bergs RA, Eberhart R, Baker L, Fernandez R and Cadeddu JA: Trocar-less instrumentation for laparoscopy: magnetic positioning of intra-abdominal camera and retractor. Ann Surg 2007; 245: 379. 